Cite as:

Yuning He and Misty Davies.

"Validating an Air Traffic Management Concept of Operation Using Statistical Modeling."

Proceedings of the 2013 AIAA Modeling and Simulation Technologies Conference, Boston, MA.

August, 2013.

\title{
Validating an Air Traffic Management Concept of Operation using Statistical Modeling
}

\author{
Yuning $\mathrm{He}^{*}$ \\ NASA Ames University Affiliated Research Center, Moffett Field, CA, 94035, USA \\ Misty Davies ${ }^{\dagger}$ \\ NASA Ames Research Center, Moffett Field, CA, 94035, USA
}

\begin{abstract}
Validating a concept of operation for a complex, safety-critical system (like the National Airspace System) is challenging because of the high dimensionality of the controllable parameters and the infinite number of states of the system. In this paper, we use statistical modeling techniques to explore the behavior of a conflict detection and resolution algorithm designed for the terminal airspace. These techniques predict the robustness of the system simulation to both nominal and off-nominal behaviors within the overall airspace. They also can be used to evaluate the output of the simulation against recorded airspace data. Additionally, the techniques carry with them a mathematical value of the worth of each prediction-a statistical uncertainty for any robustness estimate. Uncertainty Quantification (UQ) is the process of quantitative characterization and ultimately a reduction of uncertainties in complex systems. UQ is important for understanding the influence of uncertainties on the behavior of a system and therefore is valuable for design, analysis, and verification and validation. In this paper, we apply advanced statistical modeling methodologies and techniques on an advanced air traffic management system, namely the Terminal Tactical Separation Assured Flight Environment (T-TSAFE). We show initial results for a parameter analysis and safety boundary (envelope) detection in the high-dimensional parameter space. For our boundary analysis, we developed a new sequential approach based upon the design of computer experiments, allowing us to incorporate knowledge from domain experts into our modeling and to determine the most likely boundary shapes and its parameters. We carried out the analysis on system parameters and describe an initial approach that will allow us to include time-series inputs, such as the radar track data, into the analysis.
\end{abstract}

\section{Introduction}

NASA is developing new tools and procedures that are intended to improve the safety of the nation's air transportation system and to replace the legacy conflict prediction tools in place today. One promising NASA concept of operation, the Tactical Separation Assured Flight Environment (TSAFE), works in the en route airspace and uses both flight intent information and dead reckoning to calculate trajectories. ${ }^{1,2}$ TSAFE was specifically designed for conflict detection en route, and is part of a larger airspace management system known as the Automated Airspace Concept. ${ }^{3}$ Detecting conflicts within the terminal airspace can be more complex than detection within en route airspace, for reasons we will discuss below. NASA has developed a tactical conflict detection and resolution tool specifically designed for the complexities of the terminal airspace that is based on TSAFE, called the Terminal Tactical Separation Assured Flight Environment, or Terminal TSAFE (T-TSAFE). ${ }^{4}$

An important safety goal we focus on in this work is to maintain safe separation between all aircraft. Maintaining separation in the terminal airspace (near an airport) is much more challenging than maintaining separation in en route airspace. In the terminal airspace there are higher rates of operational errors, denser

\footnotetext{
*Assistant Research Scientist, Intelligent Systems Division, Mail Stop 269-1, AIAA Member.

${ }^{\dagger}$ Research Computer Engineer, Intelligent Systems Division, Mail Stop 269-1, AIAA Senior Member.
} 
air traffic, frequent large turns, incompletely specified flight plans, complex sets of separation standards, and the frequent necessity to operate aircraft purposely near the required separation standards. Terminal airspace surrounds airports within a radius of about 40 miles. Within terminal airspace, the legal separation standards are complex, and depend on aircraft weight class, type of approach, visual vs. instrument flight rules, and whether the aircraft is transitioning to or from en route airspace. Terminal area air traffic controllers guide aircraft as they approach or depart the airport, and must maintain these separation standards. However, the complexity of terminal airspace has proven difficult for the tactical conflict detection systems that are intended to aid terminal area air traffic controllers.

The Terminal Tactical Separation Assured Flight Environment (T-TSAFE) that is being developed at NASA Ames is designed to overcome these difficulties. T-TSAFE creates a single predicted trajectory for each aircraft based on an algorithm that uses available flight intent information. The flight intent information includes flight plans, area navigation departure routes, site-specific nominal arrival routes, speed restrictions, and altitude clearances. Additionally, the separation algorithms in T-TSAFE are governed by a large number of configuration, aircraft performance (e.g., aircraft weight), and operational parameters. T-TSAFE also includes a refined set of current, dynamic separation standards for terminal airspace to define losses of separation. By combining all of these variables, T-TSAFE is able to predict the future positions of aircraft and check them for possible conflicts with significantly fewer false alerts than the current legacy system. ${ }^{4}$

Obviously, it is important to ensure T-TSAFE is working correctly and produces as few prediction mistakes as possible, even under off-nominal conditions or when the normal behavior of the air traffic system changes. Our goal is to work towards the validation of T-TSAFE within this safety-critical system. In

particular, we want to a.) make sure that the system is robust to extreme input configurations and also to b.) quantify the effect of uncertainties in the system on the predictions. In order to ensure safe operation through validation, we must analyze how uncertainties and noise in those parameters influence the behavior of T-TSAFE. However, current validation techniques for such systems are usually limited to exploring a small set of manually selected individual senarios.

Uncertainty Quantification (UQ) is an active area of science that seeks to understand the sources of prediction error in a complex system, and further to quantify and to hopefully reduce these sources of uncertainty in order to achieve better predictions about the complex system's behavior. ${ }^{5,6}$

UQ is important for understanding the influence of uncertainties in the behavior of the system and therefore is very valuable for design, analysis and, in particular for the focus of our work, validation of the system. In order to carry out a UQ analysis for T-TSAFE, it is important to understand interactions between continuous variables (variables which can have infinitely small variation within their range), discrete variables (variables which can only take on 1 of $n$ finite values), and time series variables (variables which can change their values with time). Systems which depend on a combination of continuous and discrete variables for their behavior are known as hybrid systems and require a substantial extension to the current state of the art for a complete analysis. ${ }^{7,8}$ In this paper, we carry out an initial UQ analysis for T-TSAFE; our initial results give insight about those configuration parameters which are actually influential on T-TSAFE's capability to detect losses of separation between two aircraft.

\section{A. Separation Assurance}

The National Airspace (NAS) is at its performance limit and the number of flights may increase by a factor of 2 or more by the year 2020. ${ }^{9,10}$ Researchers on Next Generation Air Traffic Control develop approaches and systems to safely handle the increased number of aircraft in the NAS by using increasingly automated systems. The important safety goal is to maintain safe separation between all aircraft: usually $3 \mathrm{nmi}$ horizontal and 1000 feet vertical, referred to as "the hockey puck" (see Figure 1). The terminal airspace near an airport is much more challenging in terms of density and complexity than en route airspace, and operating errors are higher. The complexity of terminal airspace has proven difficult for tactical conflict detection systems. Contributing factors to this complexity include the dense air traffic, frequent large turns, incompletely specified flight plans, a complex set of separation standards, and the frequent necessity to operate aircraft purposely near the required separation standards.

Accurate and early prediction of any loss of separation (LOS) is necessary to support the controller or for automated systems. There are three independent layers of separation assurance. The strategic layer focuses on mid-term conflicts (losses of separation) predicted to be between 2 and 20 minutes into the future. The tactical layer addresses short-term or imminent conflicts predicted to occur within 2 minutes. A third layer of safety is provided by an independent airborne collision avoidance system such as TCAS (Traffic Alert 


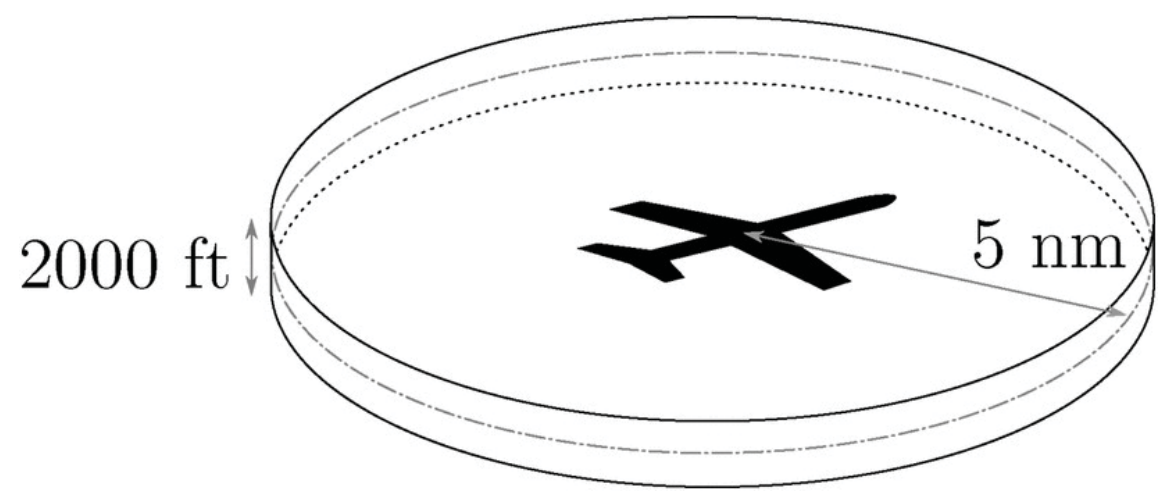

Figure 1. The aircraft separation "hockey-puck"11

and Collision Avoidance System). TCAS deals with potential collisions less than 45 seconds away. The tactical layer for en route flights, known as the Tactical Separation Assured Flight Environment (TSAFE), is proposed as a backup system that duplicates a limited set of safety-critical functions of the strategic layer. TSAFE simplifies the problem of automated separation assurance and provides a safety net for the strategic layer. Algorithms for the prediction of LOS on all layers are safety-critical and thus require careful analysis and validation.

\section{B. Details of the T-TSAFE System}

The Terminal Tactical Separation Assured Flight Environment (T-TSAFE) predicts LOS near airports for take-offs and landings. ${ }^{12}$ T-TSAFE encodes all complex terminal separation rules including wake separation. T-TSAFE uses a novel intent-based algorithm for predicting the trajectories of the aircraft for the next 2 minutes and is implemented in the Java computer language.

The architecture of T-TSAFE is shown in Figure 2. T-TSAFE uses different kinds of inputs: static configuration data (TSAFE properties), static aircraft data (e.g., aircraft weight, climb speed, and other performance data as given in the Base of Aircraft Data (BADA $)^{13,14}$ ), and the actual aircraft track data including weather information. Track data and weather are time series data. Track data, stored in CTAS's cm-sim data format contain the aircraft's ID and flight number, position, ground speed, altitude, and additional data in time intervals of 12 seconds (one ATC radar sweep). The weather data contain wind speed, direction, temperature, and air pressure for a certain location in a $5 \mathrm{~km}$ grid and altitude for each hour of the day.

After reading the static data, T-TSAFE processes the track data and reports potential conflicts with time-to-loss (ttlos) and performs predictions in regular intervals. A utility function read_conflicts reads those reported conflicts and produces a data structure for further processing.

Figure 3 shows a typical scenario with two aircraft. The track data originate from an actual LOS scenario in which we have obscured the airline information. The aircraft track for flight ABC653 is shown in blue, for XYZ1052 in green. Both aircraft are in final descent. The figure shows the world coordinates in nautical miles (nmi) in the $\mathrm{x}$ and $\mathrm{y}$ directions and the altitude in thousands of feet. The red lines connect the trajectories at points in which there were actual losses of separation; when both aircraft came too close together and violated the terminal separation rules. This figure shows that several losses of separation are detected (numlos); the first loss of separation occurs at time ttlos.

\section{Statistical Modeling for T-TSAFE}

In our study, we investigated how T-TSAFE copes with uncertainties and trajectory prediction (TP) errors. In general, uncertainties can occur due to:

- intent errors and delays (e.g. pilot doesn't follow ATC commands),

- errors in the initial conditions (e.g. radar measurements), 


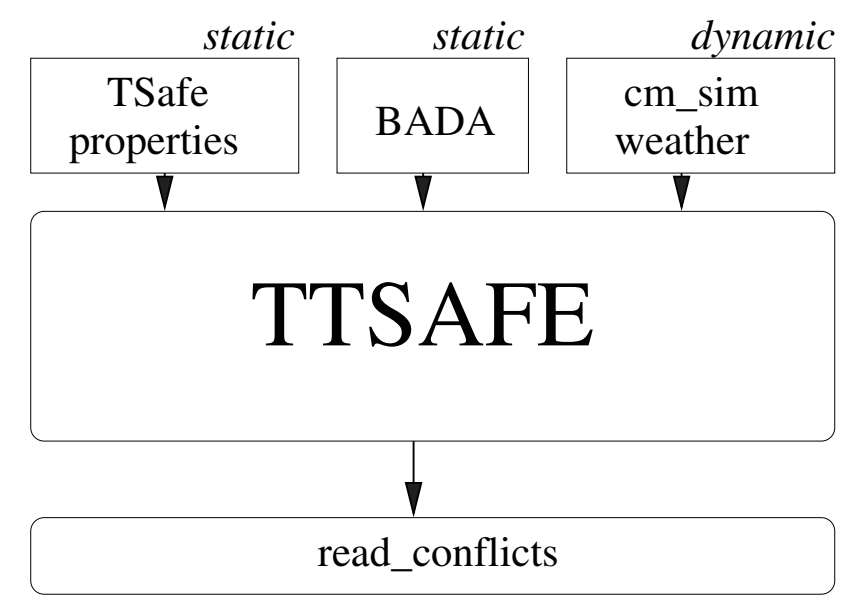

Figure 2. Architecture of TTSAFE

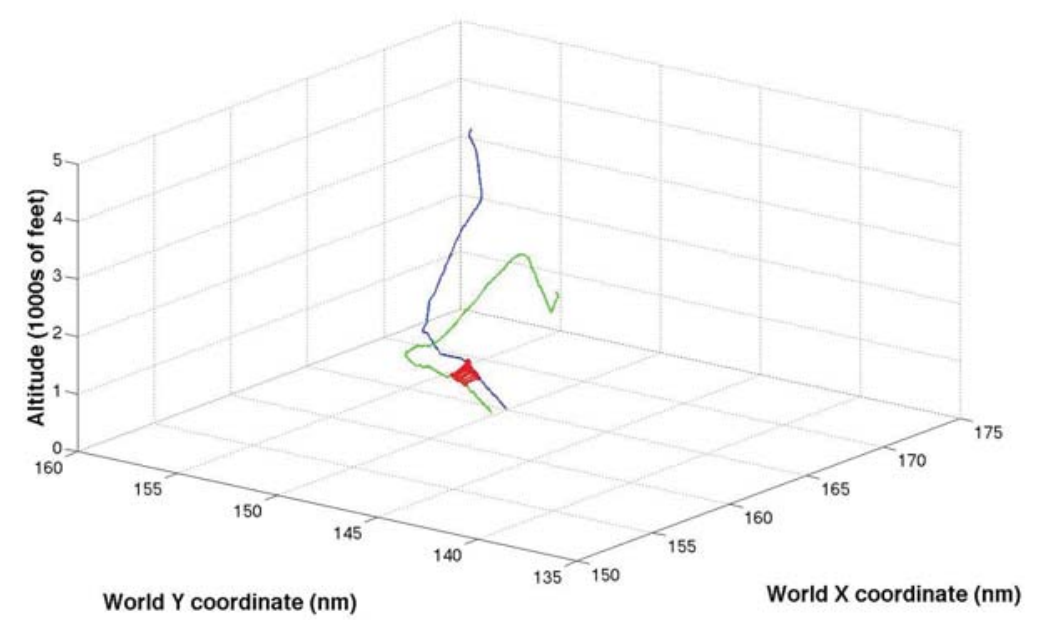

Figure 3. Two aircraft scenario, points at which the aircraft lose separation are connected with red lines.

- environmental uncertainties (e.g. weather),

- and modeling errors (e.g. unknown actual weight of aircraft).

We analyzed how the specific separation assurance algorithm in T-TSAFE behaves in the presence of these uncertainties. In general, we consider that all three kinds of inputs to T-TSAFE as shown in Figure 2 can contain sources of uncertainties. However, in this paper we report only on the uncertainty with respect to the configuration inputs and to the track data. We will present a more thorough analysis in future work. We perform both time series analysis and boundary analysis within our study, using techniques only recently developed within the statistics community. ${ }^{15}$ In the following section, we first give a short introduction into Uncertainty Quantification (UQ). We then overview sensitivity analysis and briefly discuss our boundary estimation technique. Finally, we describe and report on our experimental in the subsequent sections. 


\section{Uncertainty Quantification}

\section{A. Concepts}

Uncertainty Quantification(UQ) is the science of quantitative characterization and reduction of uncertainties in systems. UQ is increasingly becoming an important part of design and analysis of complex computer and engineering systems. ${ }^{16-18}$ It provides the ability to assess the quality of computational results and apply confidence bounds to output metrics, particularly problems in which experimental data is difficult or impossible to obtain.

Uncertainties from numerous sources can affect the behavior of a complex system, such as:

- Parameter uncertainty, which comes from the model parameters that are inputs to the computer model, but whose exact values are unknown to experimentalists and cannot be controlled in physical experiments;

- Structural uncertainty, aka model inadequacy, model bias, or model discrepancy, which comes from the lack of knowledge of the underlying true physics. It depends on how accurately a mathematical model describes the true system for a real-life situation, considering the fact that models are almost always only approximations to reality;

- Algorithmic uncertainty, aka numerical uncertainty, which comes from numerical errors and numerical approximations per implementation of the computer model. Most models are too complicated to solve exactly;

- Experimental uncertainty (observation error) which comes from the variability of experimental measurements. UQ is valuable for design analysis and for validation.

In general, there are two types of problems in UQ analysis: forward uncertainty propagation is used for the analysis of system outputs based on uncertain inputs; the inverse problem is used to assess model and parameter uncertainty based on the outputs.

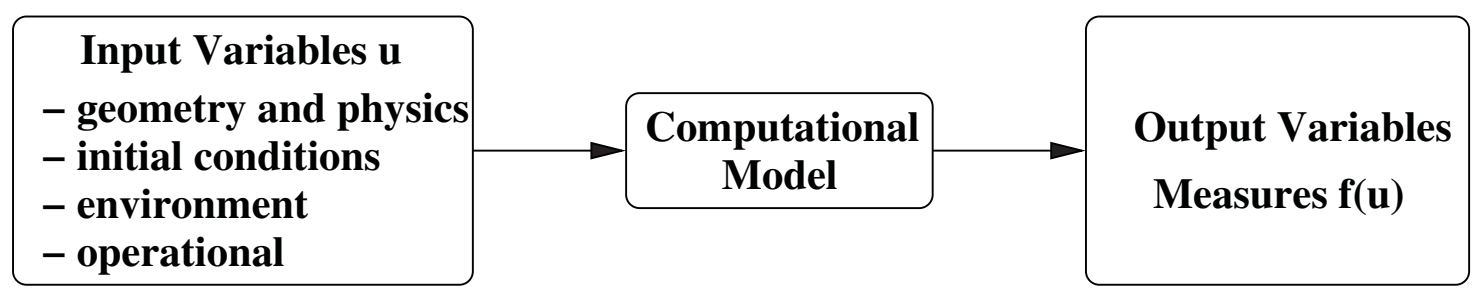

Figure 4. Uncertainty Quantification

\section{B. Sensitivity Analysis}

For the parameter analysis, we are interested in studying how the uncertainty in the output of the system can be apportioned to different sources of uncertainty in its inputs. To do so, we performed sensitivity analysis on the parameters, and usually uncertainty and parameter sensitivity analysis are run in tandem. Sensitivity analysis can be useful for a range of purposes, some examples include:

- Testing the robustness of the system in the presence of uncertainty,

- Identifying model inputs that cause significant uncertainty in the output thus should therefore be the focus of further analysis,

- Model simplification i.e. fixing model inputs that have no effect on the output,

- Efficient searching for errors in the model.

In models involving many input variables, parameter analysis is an essential ingredient of model building and quality assurance. 


\section{Experiments and Results}

\section{A. Experimental Setup: T-TSAFE Harness}

For our statistical analysis, we consider T-TSAFE strictly as a black box, i.e., we do not have access to any of the internal details and variables of the T-TSAFE system. For each experimental trial, we provide TTSAFE with perturbed variables to its configuration parameters and to the track data, as shown in Figure 2. Each of the inputs is parameterized, i.e., it can deviate in a certain way from its original, or nominal value. Table 1 gives the values perturbed for the static configuration and we discuss our track perturbation below in subsection C. As the driving scenario, we use - for this study - the two-aircraft scenario shown in Figure 3: flights ABC653 and XYZ1052 losing safe separation during their descent.

Figure 5 shows our experimental setup. In a first step, the variables and parameters that need to be perturbed (see above) must be specified by the user. Typically, a range can be given, e.g., the time interval for invoking the detection algorithm is between 5 seconds and 200 seconds, or variations around the nominal value, e.g., the aircraft weight $w$ can be perturbed by $\pm 25 \%$, i.e., $w=w_{0} \pm 25 \%$.

Based upon this specification, our harness generates a number of test cases. Each test case corresponds to a specific set of inputs. With those inputs the TTSAFE system is started, and the outputs of interest, mainly the variables numlos, ttlos and the position and altitude of the first detection of LOS $(x, y, a l t)$ are extracted using the utility function read_conflicts. After all relevant test cases have been processed, we perform the statistical data analysis and visualize the results.

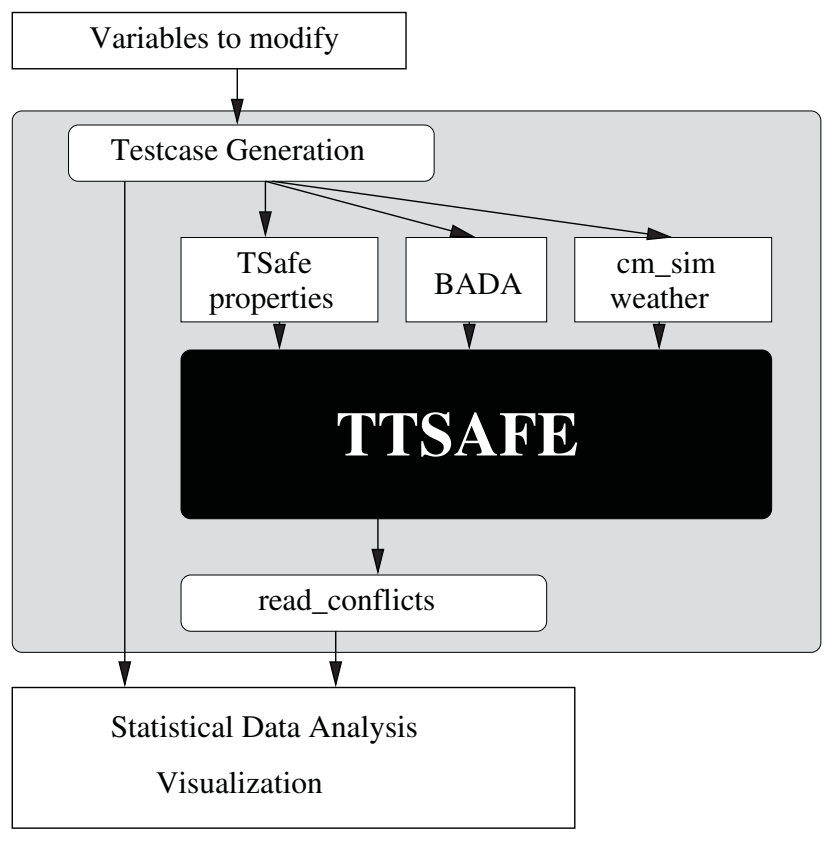

Figure 5. Architecture of the TTSAFE Harness

\section{B. Sensitivity Analysis on Configuration Inputs}

For the analysis on configuration inputs, we used 14 scalar configuration parameters as shown in Table 1. Of those, 10 are continuous and 4 variables are discrete. With this parameter definition, we used a 3-factor combinatorial exploration ${ }^{19-21}$ to generate 2467 distinct test cases. For continuous variables, a uniform distribution within a range of $\pm 25 \%$ was approximated using 5 equidistant bins. The discrete configuration parameters are used to select between varying algorithms for detecting conformance and predicting aircraft trajectories. We executed T-TSAFE on each of the 2467 test cases and we recorded numlos and ttlos.

For the given experiments and each of the parameter variables, we calculated the standardized regression coefficient (SRC), a commonly used sensitivity value. Figure 6 shows the corresponding plot. Small circles show the SRC values, the short lines indicate their variance (which is very low in this case). This plot 


\begin{tabular}{|l|l|c|}
\hline Parameter Name & Nominal Value & Type \\
\hline \hline MinHorizSepNmi & 3 & $\mathrm{C}$ \\
aboveFL290AltLevel & 1000 & $\mathrm{C}$ \\
aboveFL290AltChanging & 1000 & $\mathrm{C}$ \\
belowFL290AltLevel & 1000 & $\mathrm{C}$ \\
belowFL290AltChanging & 1000 & $\mathrm{C}$ \\
CheckPeriodMinutes & 1.0 & $\mathrm{C}$ \\
CrossTrackTolerance & 0.5 & $\mathrm{C}$ \\
AlongTrackTolerance & 0.25 & $\mathrm{C}$ \\
AltitudeTolerance & 200 & $\mathrm{C}$ \\
HeadingTolerance & 5 & $\mathrm{C}$ \\
ConformanceStrategy & No default & $\mathrm{D}(2)$ \\
ConflictDetection & No default & $\mathrm{D}(3)$ \\
NomTrajSynth & No default & $\mathrm{D}(3)$ \\
DeadReckonTS & No default & $\mathrm{D}(2)$ \\
\hline \hline
\end{tabular}

Table 1. TTSAFE configuration parameters perturbed in our experiments. Continuous parameters (C) were perturbed with a range of $\pm 25 \%$ of the nominal value. $\mathbf{D}(x)$ denotes a discrete variable with $x$ distinct values.

indicates that only three variables ( MinHorizSepNmi, CheckPeriodMinutes, and ConflictDetection) have SRC values which are substantially larger than 0 , meaning that the behavior of T-TSAFE is sensitive to those variables.

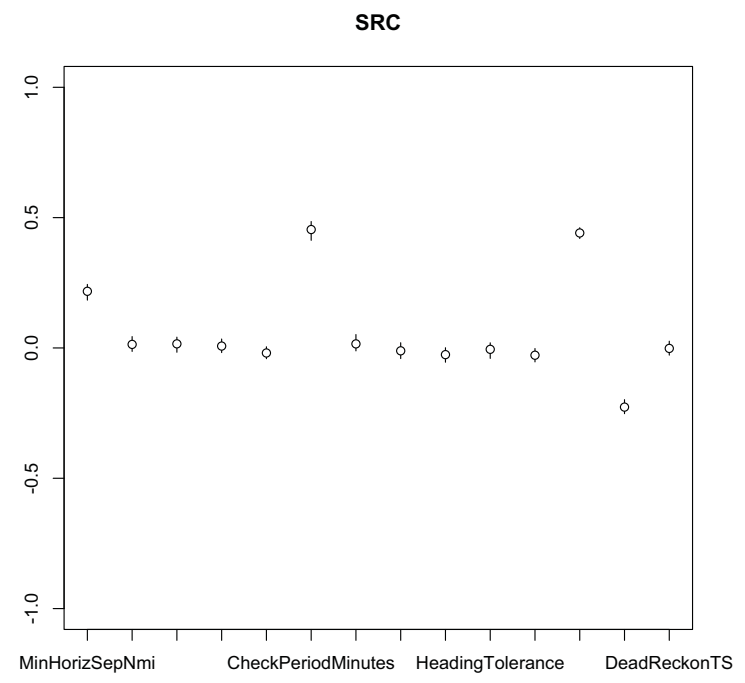

Figure 6. Sensitivity Analysis Results. Values near zero indicate that the output is insensitive to the input.

Some of the results make sense. For example, MinHorizSepNmi (the minimum allowable horizontal separation in nautical miles) governs if there is a LOS or not, so T-TSAFE should be sensitive to that input. Other results, such as the fact that T-TSAFE is relatively insensitive to the algorithms used for trajectory prediction, are less intuitive. It may be that a more sophisticated exploration in which the trajectories of the aircraft are significantly varied would produce a different result. Figure 7 shows the scatter plots for numlos, supporting the results of the sensitivity analysis. 

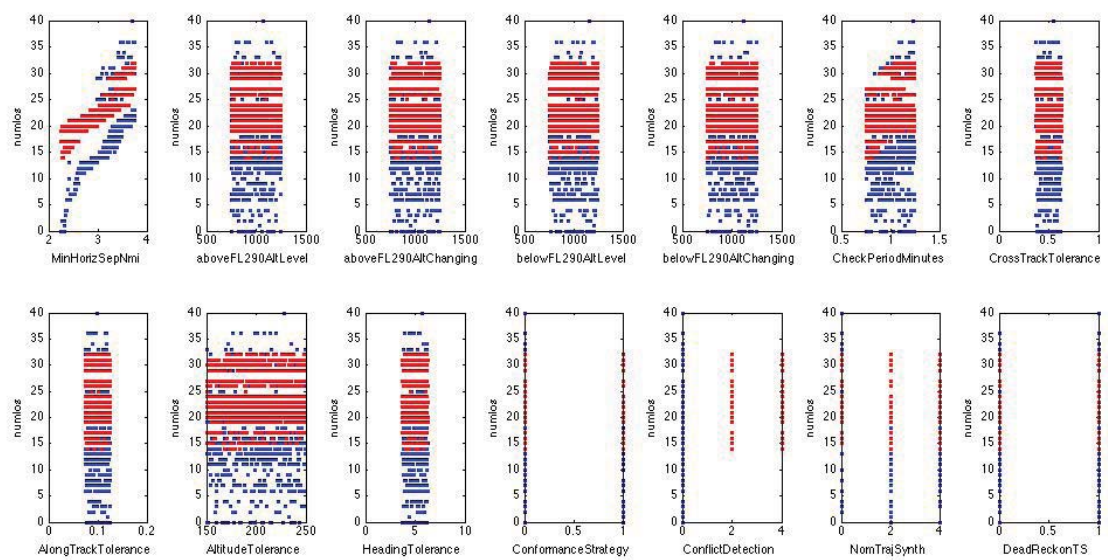

Figure 7. Scatterplots for numlos

\section{Uncertainty in Track Data}

For this experiment, uncertainties in the measured track data of both aircraft were analyzed. These track data are generally obtained by measurements of ATC radar and can be sources of substantial errors and uncertainties.

These data are time series data and a number of different possibilities exist to perturb them for uncertainty analysis. Here, we pick a simple, yet relevant mechanism: each of the track data are offset by a given, fixed bias. Such a perturbation can, for example, correspond to a systematic bias in the radar-based altitude measurements.

More specifically, for this experiment, we modified the altitude by a uniformly distributed time-constant bias $\delta \in\{-1000 \mathrm{ft}, 1000 \mathrm{ft}\}$ for each aircraft. Figure 8 shows the behavior of TTSAFE over the bias $\delta_{A C 1}$ and $\delta_{A C 2}$. The nominal case is thus in the middle of this figure. Individual trials are shown in magenta for numlos $<4$ (i.e., no losses detected), red for $4 \leq$ numlos $<7$, and blue otherwise. Obviously the triangular shapes are caused by the geometry of the scenario: if the bias causes the aircraft be too far away from each other vertically, then fewer losses are detected. The asymmetry between AC1 and AC2 might be of interest for further investigation as well as a "red" region surrounded by blue in the vicinity of $\delta_{A C 1}=-800 \ldots-300$ and $\delta_{A C 2}=-200 \ldots 200$.

A similar visualization for ttlos is shown in Figure 9. The same trials as discussed above show quite a different picture: ttlos keeps its nominal value except for a tiny area in the parameter space. Trials are marked in red for ttlos $<0.5$.

This small (appr. 125x125ft) area could be of interest for design and validation as it shows a high sensitivity and is located in the "middle" of an otherwise smooth region. A zoomed-in plot is shown in Figure 9 (right), revealing a triangular shape.

\section{Conclusions}

In this paper, we show an initial uncertainty quantification analysis for an air traffic management concept of operation (T-TSAFE). This analysis shows that there is a wide variation in the effect the input configuration parameters have on the ability of T-TSAFE to detect losses of separation, as seen in Figure 6. It also revealed an unexpected region of behavior that can be affected by an altitude measurement bias, as shown in Figure 8. We believe that these sorts of analyses are invaluable to the design and analysis of complex systems, particularly those that are safety-critical.

In our future work, we plan to:

- extend current methodologies and perform further analysis for hybrid inputs consisting of continuous and discrete parameters, 


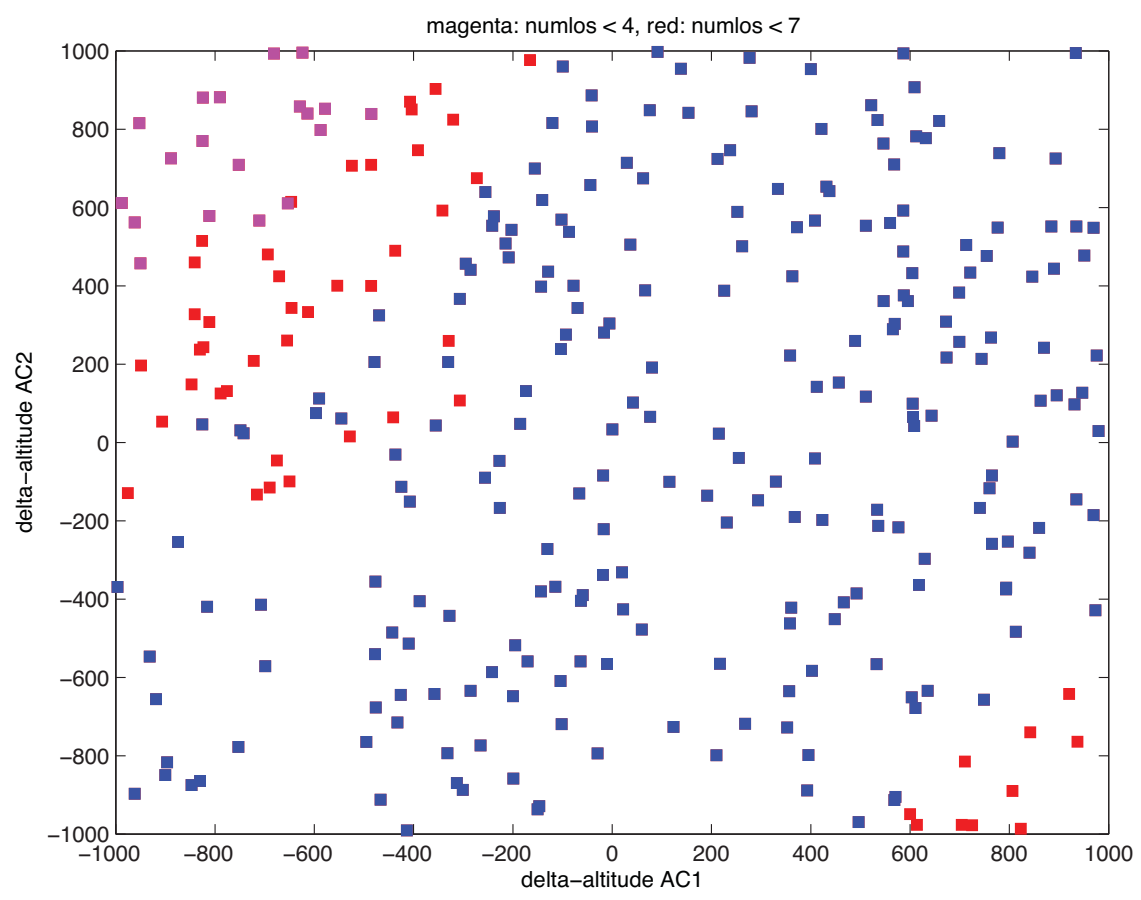

Figure 8. Different values of numlos over different altitude biases for AC1 and AC2

- use this extended analysis technique to study additional TTSAFE outputs - e.g. estimated points of LOS, aircraft conformance,

- perform uncertainty analyses for aircraft parameters such as weight and performance, e.g., study the influence of weight on climb and descent speed,

- further study the influence of track uncertainty (time-series data),

- use multi-aircraft scenarios for the analysis of missed-alarm/false-alarm rates.

\section{Acknowledgments}

This research was conducted at NASA Ames Research Center. Reference herein to any specific commercial product, process, or service by trade name, trademark, manufacturer, or otherwise, does not constitute or imply its endorsement by the United States Government.

\section{References}

\footnotetext{
${ }^{1}$ Tang, H., Denery, D., Erzberger, H., and Paielli, R., "Tactical Separation Algorithms and Their Interaction with Collision Avoidance Systems," American Institute of Aeronautics and Astronautics (AIAA) Guidance, Navigation, and Control (GNC) Conference and Exhibit, Honolulu, HI, 18-21 Aug., 2006.

${ }^{2}$ Paielli, R., "Tactical Conflict Resolution Using Vertical Maneuvers in Enroute Airspace," American Institute of Aeronautics and Astronautics (AIAA) Aviation Technology, Integration, and Operations (ATIO) Conference, Anchorage, AK, USA, Sep. 2008.

${ }^{3}$ Erzberger, H., "The Automated Airspace Concept," 4th USA/Europe Air Traffic Management RED Seminar, Santa Fe, NM, USA, Dec. 2001.

${ }^{4}$ Tang, H., Robinson, J., and Denery, D., "Tactical Conflict Detection in Terminal Airspace," Journal of Guidance, Control, and Dynamics, Vol. 34, No. 2, 2011, pp. 403-413.

${ }^{5}$ Lee, S. and Chen, W., "A comparative study of uncertainty propagation methods for black-box-type problems," Structural and Multidisciplinary Optimization, Vol. 37, No. 3, 2009, pp. 239-253.
} 

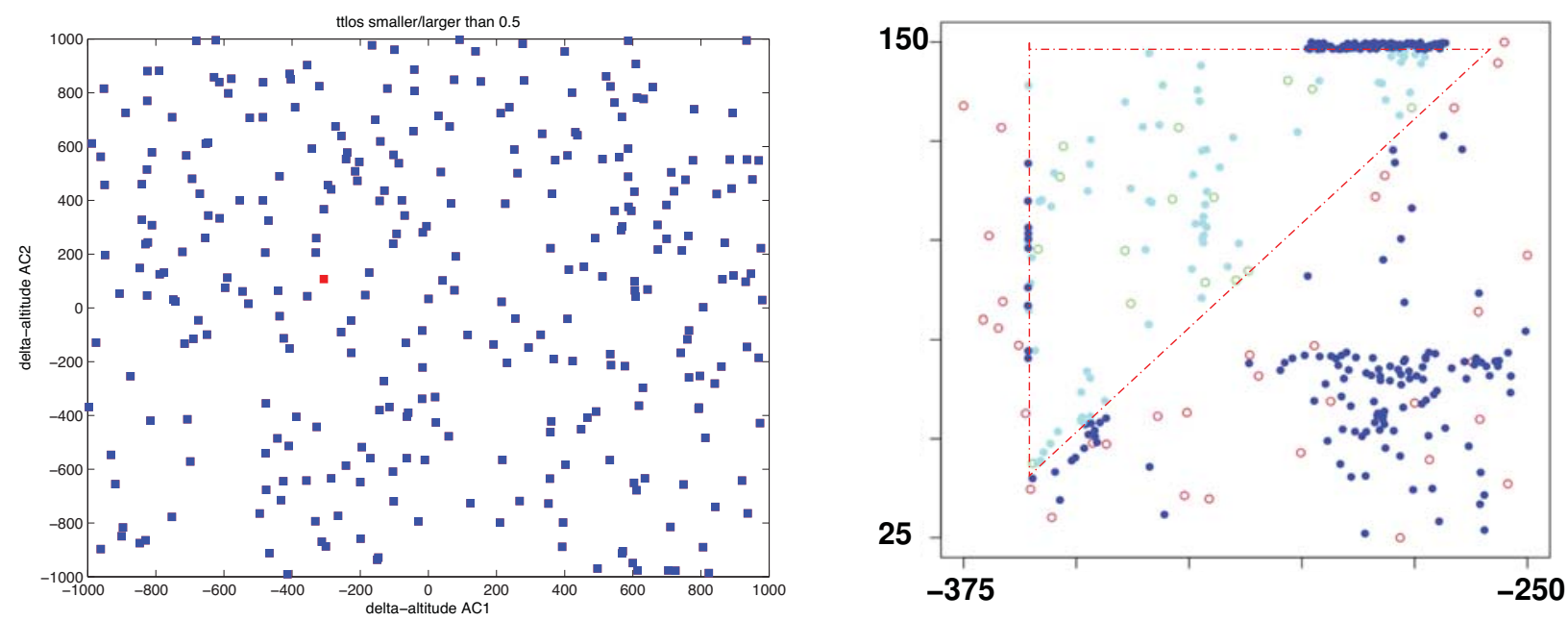

Figure 9. Left: ttlos over altitude perturbations for aircraft 1 and aircraft 2. Blue dots correspond to ttlos $>$ 0.5, red to ttlos $<0.5$. Right: Blown up small range as obtained by the active learning algorithm. Blue dots correspond new data points with ttlos $>0.5$; cyan dots to ttlos $<0.5$.

${ }^{6}$ Matthies, H., "Quantifying Uncertainty: Modern Computational Representation of Probability and Applications," Extreme Man-Made and Natural Hazards in Dynamics of Structures, edited by A. Ibrahimbegovic and I. Kozar, NATO Security through Science Series, Springer Netherlands, 2007, pp. 105-135.

${ }^{7}$ Henzinger, T. A., "The Theory of Hybrid Automata," 11th Annual Symposium on Logic in Computer Science (LICS), 1996, pp. 278-292.

${ }^{8}$ Goebel, R., Sanfelice, R. G., and Teel, A. R., "Hybrid dynamical systems," IEEE Control Systems Magazine, Vol. 29, No. 2, 2009, pp. 28-93.

${ }^{9}$ Erzberger, H., "Transforming the NAS: The next generation air traffic control system," 24th International Congress of the Aeronautical Sciences, Yokohama, Japan, 2004.

10 "Next Generation Air Transportation System Integrated Plan," December 122004.

${ }^{11}$ Ellerbroek, J., Visser, M., Van Dam, S. B. J., Mulder, M., and Van Paassen, M., "Design of an Airborne ThreeDimensional Separation Assistance Display," Systems, Man and Cybernetics, Part A: Systems and Humans, IEEE Transactions on, Vol. 41, No. 5, 2011, pp. 863-875.

${ }^{12}$ Tang, H., Robinson, J., and Denery, D., "Tactical Conflict Detection in Terminal Airspace," 10th AIAA Aviation Technology, Integration, and Operations (ATIO) Conference, 2010.

${ }^{13}$ Eurocontrol, "Base of Aircraft Data (BADA) at http://www.eurocontrol.int/services/bada," July 2013.

${ }^{14}$ Gillet, S., Nuic, A., and Mouillet, V., "Enhancement in Realism of ATC Simulations by Improving Aircraft Behaviour Models," 29th Digital Avionics Systems Conference, Oct. 2010.

${ }^{15} \mathrm{He}, \mathrm{Y} .$, Variable-length Functional Output Prediction and Boundary Detection for an Adaptive Flight Control Simulator, Ph.D. thesis, 2012.

${ }^{16}$ Chen, W., Jin, R., and Sudjianto, A., "Analytical Variance-Based Global Sensitivity Analysis in Simulation-Based Design Under Uncertainty," ASME Journal of Mechanical Design, Vol. 127, 2005, pp. 875-886.

${ }^{17}$ Helton, J., Johnson, J., Oberkampf, W., and Sallaberry, C., "Sensitivity analysis in conjunction with evidence theory representations of epistemic uncertainty," Reliability Engineering System Safety, Vol. 91, No. 1011, 2006, pp. 1414 - 1434.

${ }^{18}$ Helton, J. C. and Johnson, J. D., "Quantification of margins and uncertainties: Alternative representations of epistemic uncertainty," Reliability Engineering System Safety, Vol. 96, No. 9, 2011, pp. 1034 - 1052.

${ }^{19}$ Schumann, J., Gundy-Burlet, K., Pasareanu, C., Menzies, T., and Barrett, T., "Software V\&V Support by Parametric Analysis of Large Software Simulation Systems," Proc. IEEE Aerospace, IEEE Press, 2009.

${ }^{20}$ Gundy-Burlet, K., Schumann, J., Menzies, T., and Barrett, T., "Parametric Analysis of ANTARES Re-entry Guidance Algorithms Using Advanced Test Generation and Data Analysis," Proc. iSAIRAS 2008 (9th International Symposium on Artifical Intelligence, Robotics and Automation in Space), 2008.

${ }^{21}$ Schumann, J., Gundy-Burlet, K., Pasareanu, C., Menzies, T., and Barrett, T., "Tool Support for Parametric Analysis of Large Software Systems," Automated Software Engineering, 23rd IEEE/ACM International Conference, 2008. 